\title{
Influence of Varying Doses and Duration of Dietary Nitrates from Beetroot Juice on Sprint Performance in U.S. Army ROTC Cadets A Pilot Study
}

\author{
Michael Melton ${ }^{1}$, Jayanthi Kandiah²
}

${ }^{1}$ Department of Nutrition and Health Science, Ball State University, USA, ${ }^{2}$ Department of Nutrition and Health Science HB 214N, Ball State University, Muncie, IN - 47306, USA

Address for correspondence: Dr. Jayanthi Kandiah, Department of Nutrition and Health Science HB 214N, Ball State University, Muncie, IN - 47306, USA. Phone: 765-285-5955. E-mail jkandiah@bsu.edu

\section{Abstract}

Objective: The objective of the study was to assess the effects of varying levels and duration of dietary nitrate supplementation from beetroot juice (BR) on sprint performance in army Reserve Officer Training Corps (ROTC) cadets. Materials and Methods: Army ROTC cadets were randomly assigned to one of three treatment groups: Control (CON); low BR dose (BR1); and high BR juice-BR2. For 0, 6, and 15 days, nitrate consumption from $B R$ groups was as follows: CON received one $16.9 \mathrm{oz}$. bottle of apple juice $\left(0 \mathrm{mg} \mathrm{NO} \mathrm{NO}_{3}^{-}\right)$; $\mathrm{BR} 1$ received one can of $\mathrm{BR}$ juice $\left(300 \mathrm{mg}, 4.84 \mathrm{mmol} \mathrm{NO}{ }_{3}^{-}\right.$), and BR2 received 2 cans (16.8 oz.) BR (600 mg, $9.68 \mathrm{mmol} \mathrm{NO}_{3}-$ ). One week before the study, each cadet completed body composition measurements, predicted aerobic capacity measurements, and nutritional analysis through two 24-h dietary recalls. Differences in primary measures (distance covered in the Yo-Yo IR1) were analyzed with two-way repeated measures analysis of variance (ANOVA) tests both between groups (CON, BR1, and BR2) and within groups $(0,6$, and 15 days). Descriptive statistics and frequency counts were run on all remaining variables with a one-way ANOVA or $t$-test, including maximal heart rate during the Yo-Yo IR1, dietary compliance, daily blood pressure, juice compliance, and conditioning workouts. Results: A dose-related enhancement with BR was observed; the data trended toward significance even in this small sample. A t-test revealed that there was a significant difference in sprint performance by males and females overall at days 0,6 , and $15(P=0.025$, $P=0.005$, and $P=0.004$, respectively). Conclusion: A single ( $300 \mathrm{mg}, 4.84 \mathrm{mmol} \mathrm{NO}_{3}$ ) or double (600 mg, $9.68 \mathrm{mmol} \mathrm{NO}_{3}-$ ) daily dose consumption of BR appears to benefit ROTC cadets in athletic performance. Daily consumption of BR benefitted ROTC males more than females. Results suggest BR supplementation could be advantageous for sprint performance when administered for a longer duration (> 15 days).

\section{Keywords: Dietary nitrates, Beetroot juice, Yo-Yo intermittent recovery test level 1, Army reserve officer} training corps cadets, Varying levels and duration

\section{Background}

Consuming fruits and vegetables are encouraged as part of a healthy, balanced $\operatorname{diet}^{[1-2]}$ as they provide many health benefits such as vitamins, minerals, phytochemicals, and fiber. There is an inverse relationship between consumption of these food groups and the prevalence of adiposity. ${ }^{[3]}$ Even though most vegetables provide some dietary nitrates, ${ }^{[4-5]}$ beetroot and dark green leafy vegetables particularly are excellent sources. ${ }^{[6-7]}$
Studies have demonstrated intake of nitrates and nitrate rich foods appear to lower blood pressure, improve vasodilation, and physical performance. ${ }^{[8]}$ The effectiveness on physical performance with dietary nitrate supplementation from beetroot juice (BR), on a single day has been limited when compared to consistently acute intakes of $\geq 6$ days. ${ }^{[8]}$ Wylie et al. ${ }^{[9]}$ found among recreationally active young men, there was improvement in distance

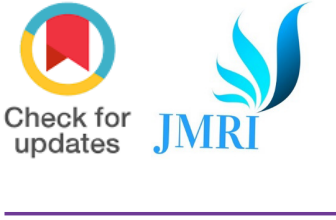

How to cite this article: Melton M, Kandiah J. Influence of Varying Doses and Duration of Dietary Nitrates from Beetroot Juice on Sprint Performance in U.S. Army ROTC Cadets A Pilot Study. J Med Res Innov. 2020;4(1):e000192.

DOI: 10.32892/jmri.192

Publication history: Received: 26-08-2019 Accepted: 21-12-2019 Published: 28-12-2019

Editor: Dr. Varshil Mehta

Copyright: Melton M, Kandiah J, This is an open-access article distributed under the terms of the Creative Commons Attribution License CC-BY 4.0., which permits unrestricted use, distribution, and reproduction in any medium, provided the original author and sources are credited.

Funding: NIL

Conflict of Interest: NIL

MK Medkrux 


\section{Melton and Kandiah: Influence of varying doses and duration of dietary nitrates from beetroot juice on sprint performance in U.S. army ROTC cadets: A pilot study}

covered after only $30 \mathrm{~h}$ of consuming BR $(490 \mathrm{~mL}$ of concentrated BR).

Athletes and military personnel, including U.S Army Reserve Officer Training Corps (ROTC) Cadets, is constantly seeking avenues to enhance their physical performance. In sports, the enhancement consists of facilitation of getting to the ball or crossing the finish line a split second faster than the opponent does. In war, it may mean overpowering the adversary and enduring the conflict. Ergogenic aids and supplements have the potential to help these populations as a performance enhancer, however, the efficacy of most have yet to be confirmed. ${ }^{[10]}$ Since modern combative military personnel is seeking ergogenic aids that would contribute to physiological improvements, optimal cognition and focus, there is more emphasis to optimize nutrition, pharmaceuticals, and dietary supplements. ${ }^{[11]}$

Beetroot is a rich source of dietary nitrates and antioxidants, as well as is safe to ingest. ${ }^{[7]}$ It has been shown to be effective for improving performance and increasing time to exhaustion in endurance sports, such as cycling ${ }^{[12]}$ and running. ${ }^{[13]}$ Very few researchers have evaluated variable doses of $\mathrm{BR}^{[9,14]}$ or an advantageous duration of supplementation. Limited research has been conducted on the use of nitrates in service members. While, there is a difference between elite combative military personnel, entry-level soldiers, and those in between the two extremes of athletic ability, body composition, and overall fitness; ROTC Cadets have been shown to have a wide range of physical fitness and body compositions ${ }^{[15]}$ likely mirroring the larger grouping of military personnel. Thus, determining the impact of a dose-related physiological response and performance effects of dietary nitrate $\left(\mathrm{NO}_{3}-\right)$ supplementation from $\mathrm{BR}$ and other food sources in ROTC Cadets is warranted. ${ }^{[16-20]}$ The objective of this study was to evaluate the effect of varying levels and duration of $B R$ as a source of dietary nitrate $\left(\mathrm{NO}_{3}-\right)$ supplementation in a coed group of Army ROTC cadets at a Midwestern U.S. University on time to exhaustion using a well-established field performance test, the Yo-Yo Intermittent Recovery Test 1 (Yo-Yo IR1).

\section{Materials and Methods}

Procedures

After approval from the University's Institutional Review Board, 27 (10 females and 17 males) conditioned Army ROTC Cadets from a midsize, Midwestern University participated in this research.
One week before the start of the research, the cadets completed assessments at the University's Integrative Exercise Physiology Laboratory. Assessments consisted of body composition measurements and predicted aerobic capacity. On the day of the testing, each cadet arrived in the laboratory between 5 and 7 am. Body composition was evaluated using whole body air-displacement plethysmography, using the BOD POD version 1.69 (Body Composition System, Life Measurement Instruments, Concord, CA). Anthropometric measurements including height (standing vertically, without shoes on) and near nude weight (as part of the BOD POD procedures, with minimal compression clothing - spandex or compression shorts for men and compression shorts, and sports bra or spandex swimsuit for women) were taken. To ensure accuracy of results during lab testing, participants were asked to refrain from several activities including eating within $3 \mathrm{~h}$, using tobacco or alcohol within $24 \mathrm{~h}$ and exercising within $8 \mathrm{~h}$. Each participant continued with the BOD POD until two quality readings were achieved.

Predicted aerobic capacity measurements were collected through the submaximal YMCA cycle ergometer protocol and an electronically braked cycle ergometer (LODE, Excalibur Sport, The Netherlands). Cadets prepared for the predictive $\mathrm{VO}_{2 \max }$ assessment on the cycle ergometer with the Polar heart rate sensor chest strap (Heart Rate Sensor H1, Polar Electro Inc., Lake Success, NY) synched to a Polar wristband (Polar FT4 Heart Rate Monitor Watch, Polar Electro Inc., Lake Success, NY). The protocol followed the YMCA submaximal test which started with $3 \mathrm{~min}$ of work at $150 \mathrm{~kg}-\mathrm{m} /$ $\min (0.5 \mathrm{~kg}$ at $50 \mathrm{rpm})$ and was followed by $2-3-$ min iterations at predetermined workloads. The workloads were based on the participant's heart rate in the $3^{\text {rd }}$ min of the first 3-min interval and increased each time; lower initial heart rates received higher workloads. The goal of the test was to have two consecutive stages with the participants' heart rate between 110 beats/minute and $85 \%$ of the age-predicted maximal heart rate to establish the predicted $\mathrm{VO}_{2 \max }$. To ensure accuracy and consistency, the same researchers completed many assessments during the same session on the entire cohort of cadets. Further, the cadets had time constraints as they had to shuttle between classes and physical training. Taking these factors into consideration, the submaximal YMCA cycle ergometer protocol was instead of the standard predicted $\mathrm{VO}_{2 \text { max }}$ as it was much easier to 


\section{Melton and Kandiah: Influence of varying doses and duration of dietary nitrates from beetroot juice on sprint performance in U.S. army ROTC cadets A pilot study}

administer and required less involvement from the participants.

Once data were collected from all cadets, they were matched evenly into three groups by predictive $\mathrm{VO}_{2 \max }$. For randomization, the study used predicted $\mathrm{VO}_{2 \max }$ relative for age and sex as the determinate of cardiovascular fitness. The researcher assigned each beverage group a random number (apple beet $=1$, all apple $=2$, and all beet $=3$ ), and then used an online random number generator to determine the order of sequence placement into a treatment group. All randomization was completed before participants receiving their respective juice. There was no significant difference in treatment groups with regard to age, height, weight, body mass index, number of females, predicted $\mathrm{VO}_{2 \max }$, relative $\mathrm{VO}_{2 \max }$ for age/ sex, ACSM categorization (fit vs. unfit), or body composition. The only difference in the groups was the beverage treatment and the quantity of nitrate consumed from BR juice. The cadets in the control group (CON) received one bottle of apple $16.9 \mathrm{oz}$. apple juice ( $0 \mathrm{mg} \mathrm{NO}_{3}-$ ) every day for 15 days. The mixed group/low BR dose (BR1) received $\sim 8 \mathrm{oz}$. apple juice +1 can (8.4 oz.) BR (300 mg, $4.84 \mathrm{mmol} \mathrm{NO}_{3}-$ ), and the cadets in the high BR dose (BR2) received 2 cans (16.8 oz.) BR (600 mg, $9.68 \mathrm{mmol} \mathrm{NO}_{3}-$ ) daily for 15 days. Cadets were educated at the initial meeting by a registered dietitian nutritionist on reporting and completing two 24-h dietary recalls of all food and beverages consumed. In addition, they were provided with a list of nitrate-rich foods and were asked to avoid consuming these foods throughout the study period. Cadets were also asked to refrain from using antibacterial mouthwash and pre-workout supplements throughout the 15-day intervention. Furthermore, two 24-h dietary recalls were conducted again in the last week of the experiment on the same weekday as their previous collection. All 24-h dietary recalls were collected, verified, and analyzed by the same registered dietitian nutritionist to verify cadets dietary compliance of nitrate-rich foods by comparing the end of experiment data to their established baseline intakes. The dietary recalls were evaluated using the Nutrition Data System for Research - Nutritional Analysis Software (University of Minnesota, 2016).

As shown in Figure 1, the performance was measured by distance covered in the Yo-Yolntermittent Recovery Test Level 1 (Yo-Yo IR1). According to Krustrup et al., ${ }^{[18]}$ this test replicated in game physiological responses, allowing for meticulous examination of physical activity of team-sport athletes off the field, which served as a valid instrument. To familiarize the cadets with the Yo-Yo IR1, before starting the research, the cadets participated in the Yo-Yo IR1 test once during the program's morning physical training session. At day 0 (baseline), day 6, and day 15, all participants performed (Military Movement Drill 1; 25 m verticals, laterals, and shuttle sprints) and then a submaximal test (the first five shuttle sprints $(200 \mathrm{~m})$ of the Yo-Yo IR1 that served as both a warm up and reminder of the exercise protocol). The Yo-Yo Intermittent Recovery Test included two $20 \mathrm{~m}$ shuttle runs with a $10 \mathrm{~s}$ recovery period cued audibly and measured distance covered by the cadets before exhaustion. When a participant failed to cover the $20 \mathrm{~m}$ distance in the allotted time, they were given a warning and the second time their performance was terminated and their distance was recorded. ${ }^{[19]}$ The sprints were completed in several heats, to ensure sufficient research assistants and heart rate monitors were available.

As it takes $2.5 \mathrm{~h}$ for plasma nitrate concentration to peak, ${ }^{[17]}$ the dosing time was set for $2-2.5 \mathrm{~h}$ before alternative-scheduled, afternoon conditioning workouts, and the research testing. Cadets who missed normally-scheduled conditioning in the early morning were allowed to make it up in the afternoon so that they could train after drinking their juice. After coordinating with the participants, the researcher created three notably visible stations across the university campus (i.e., recreation center, gymnasium,

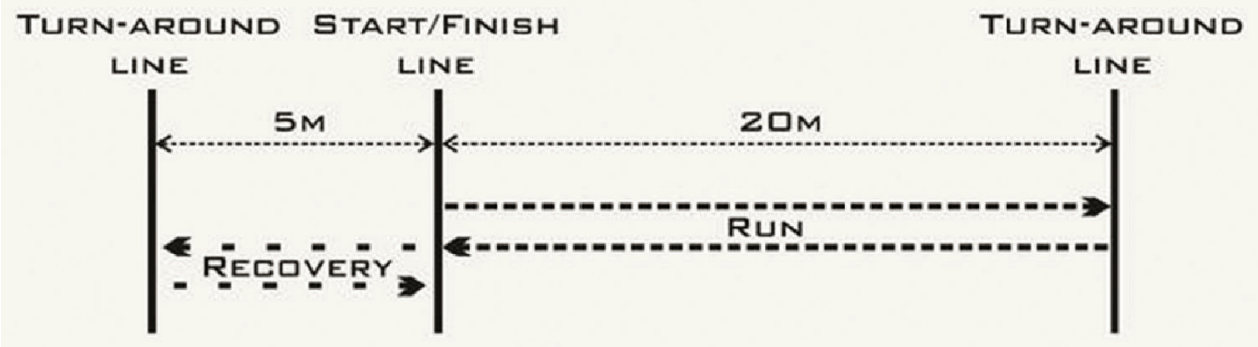

Figure 1: Diagram of Yo-Yo Test 


\section{Melton and Kandiah: Influence of varying doses and duration of dietary nitrates from beetroot juice on sprint performance in U.S. army ROTC cadets: A pilot study}

and main library) and consolidated down to one station for weekends (student center) for daily blood pressure assessments and collection of assigned juices. On arrival at all collection stations, cadets were seated for 10 min to normalize heart rate and blood pressure before blood pressure readings were evaluated using an automatic blood pressure monitor utilizing an upper arm cuff (Omron IntelliSense Professional Digital Blood Pressure Monitor, Model HEM-907XL, Omron Healthcare Inc., Lake Forest, IL). Two readings were taken with a minute between, and then the readings were averaged. After collection of blood pressure and heart rate readings, cadets received their respective beverages and were encouraged to drink all $16 \mathrm{oz}$. within $30 \mathrm{~min}$.

To ascertain cadets overall health status, information on females menstrual cycle, presence of gastrointestinal (GI) symptoms, conditioning workouts, alcohol use, food consumption practices, sleep quality, and any known injuries affecting performance. All cadets completed an online Qualtrics survey on days 8 and 15 to gather the information. Data from the survey were matched with cadets $24 \mathrm{~h}$ dietary recalls.

\section{Instruments and supplements}

The BR and apple juice used in this study was provided through a grant from Beet Performer (CAJ Food Products, Inc., Fishers, IN). CAJ Food Products was selected to provide BJ as their products came in standardized cans with known nitrate content and due to close proximity of their location for the study intervention. Each can of BR contained 8.4 fluid ounces of lacto-fermented BR, citric acid, and Vitamin B12 with no other added ingredients. This product is known commercially as Beet Performer. Each can of BR contained $300 \mathrm{mg}$ of dietary $\mathrm{NO}_{3}^{-}$(4.84 mmol) and $110 \mathrm{kcal} /$ serving ( $24 \mathrm{~g}$ carbohydrates as sugar). Each bottle of apple juice used in this study contained organic apple juice from concentrate (water, organic apple juice concentrate) with no other ingredients; this product is known commercially as Pūrjus $100 \%$ apple juice. There are $\sim 0.0 \mathrm{mg}$ of nitrates and 120 $\mathrm{kcal} / \mathrm{serving}$ ( $29 \mathrm{~g}$ carbohydrates as sugar).

\section{Statistical analysis}

Data were statistically analyzed using SPSS v. 24 for PC (2016). Differences in primary measures (distance covered in the Yo-Yo IR1) were analyzed with two-way repeated measures Analysis of variance (ANOVA) tests both between groups (CON,
BR1, and BR2) and within groups (baseline, day 6, and day 15) and their interactions. Descriptive statistics and frequency counts were run on all remaining variables with a one-way ANOVA or $t$-test, such as maximal heart rate during the Yo-Yo IR1, dietary compliance, daily blood pressure, juice compliance, conditioning workouts, alcohol and tobacco usage, fast-food consumption, sleep, and females menstrual cycle. Pearson Chi-square tests were used to analyze the frequency and intensity of Gl symptoms. Comparison between male versus female cadets along with fit versus unfit participants was added a priori. Statistical significance was set at $P \leq 0.05$. Unless otherwise stated, results are presented as mean \pm standard error.

\section{Results}

As observed in Table 1, there were no significant differences among the three groups in reference to age, body weight ( $\mathrm{kg}$, assessed by BOD POD), body mass index $\left(\mathrm{BMI}, \mathrm{kg} / \mathrm{m}^{2}\right)$, body fat percentage, or predicted relative $\mathrm{VO}_{2}(\mathrm{ml} / \mathrm{kg} / \mathrm{min})(P>0.05)$. There were no significant differences in the amount of "fit" (ACSM category good or above average for relative $\mathrm{VO}_{2 \max }$ for age and gender) and "unfit" (ACSM category average, below average, or poor for relative $\mathrm{VO}_{2 \max }$ for age and gender) among the three groups. None of the cadets were categorized as "Excellent" or "Very Poor." A one-way ANOVA for distance covered in the Yo-Yo IR1 and maximal heart rate for days 0,6 , and 15 , indicated there was no significant difference between groups $(P>0.05)$. This confirmed that even at baseline (day 0 ), before treatment, the groups were comparable.

A one-way ANOVA demonstrated that although there were slight increases in the distance covered by all three groups, this was statistically insignificant $(P=0.887)$. Further analysis revealed the CON group increased by only an additional $35 \mathrm{~m}$. However, BR1 and BR2 increased more dramatically. If the research only considered this segment of data, BR1 would have been the most suitable for performance enhancement. Results demonstrated that although all groups improved over the 15 days in sprint performance measured by distance covered, the increases were statistically insignificant $(P=0.694)$. A dose-related enhancement with BR was observed, with the data trending toward significance despite this small sample. The CON group increased by $220 \mathrm{~m}$, while BR1 increased by $276 \mathrm{~m}$ and BR2 increased by $298 \mathrm{~m}$ over the three sprints. This translates into a $25.45 \%$ increase for BR1 and a 

Melton and Kandiah: Influence of varying doses and duration of dietary nitrates from beetroot juice
on sprint performance in U.S. army ROTC cadets A pilot study

Table 1: Cadet characteristics by treatment group

\begin{tabular}{|l|c|c|c|c|}
\hline Variables & CON & BR1 & BR2 & Total \\
\hline Age (years) & $20.8 \pm 0.31$ & $19.9 \pm 0.39$ & $20.3 \pm 0.65$ & $20.3 \pm 0.27$ \\
\hline Weight $(\mathrm{kg})$ & $73.1 \pm 5.64$ & $70.7 \pm 2.12$ & $79.4 \pm 5.49$ & $74.3 \pm 2.63$ \\
\hline Body mass index $\left(\mathrm{kg} / \mathrm{m}^{2}\right)$ & $25.3 \pm 1.22$ & $24.4 \pm 1.00$ & $25.3 \pm 1.39$ & $25.0 \pm 0.67$ \\
\hline Body fat \% (decimal) & $0.22 \pm 0.03$ & $0.18 \pm 0.03$ & $0.19 \pm 0.04$ & $0.20 \pm 0.02$ \\
\hline Fat free mass (kg) & $56.6 \pm 4.02$ & $58.0 \pm 2.54$ & $64.7 \pm 6.08$ & $59.7 \pm 2.50$ \\
\hline Fat mass $(\mathrm{kg})$ & $16.5 \pm 3.18$ & $12.7 \pm 2.31$ & $14.8 \pm 2.97$ & $14.6 \pm 1.58$ \\
\hline Predicted $\mathrm{VO}_{2 \mathrm{MAx}}$ (ml/kg/min) & $44.7 \pm 2.99$ & $44.1 \pm 2.63$ & $42.1 \pm 2.18$ & $43.6 \pm 1.47$ \\
\hline Relative $\mathrm{VO}_{2 \mathrm{MAX}}$ (relative for age and sex) & $53.8 \pm 6.46$ & $50.6 \pm 6.59$ & $46.9 \pm 6.88$ & $50.4 \pm 3.72$ \\
\hline Male & 4 & 6 & 5 & 15 \\
\hline Female & 4 & 3 & 3 & 10 \\
\hline Fit & 4 & 3 & 3 & 10 \\
\hline Unfit & 4 & 6 & 5 & 15 \\
\hline
\end{tabular}

CON: Control group ( $0 \mathrm{mg} \mathrm{NO}_{3}-$ ), BR1: Low BR group ( $300 \mathrm{mg} \mathrm{NO}_{3}-$ ), BR2: High BR group ( $600 \mathrm{mg} \mathrm{NO}{ }_{3}^{-}$). Age, weight (from BOD POD, BMI, body fat, fat free mass, fat mass, predicted $V_{2_{\text {max }}}$, relative $V_{O_{2 \max }}$ presented as mean $\pm S E$. Male, female, fit (ACSM category "good" or "above average" for predicted $\mathrm{VO}_{2 \max }$ relative for age and gender), and unfit (ACSM category "average," "below average", or "poor" for predicted $\mathrm{VO}_{2 \max }$ relative for age and gender) presented as total $(n=25)$

Table 2: Differences in sprint performances between groups (CON, BR1, and BR2)

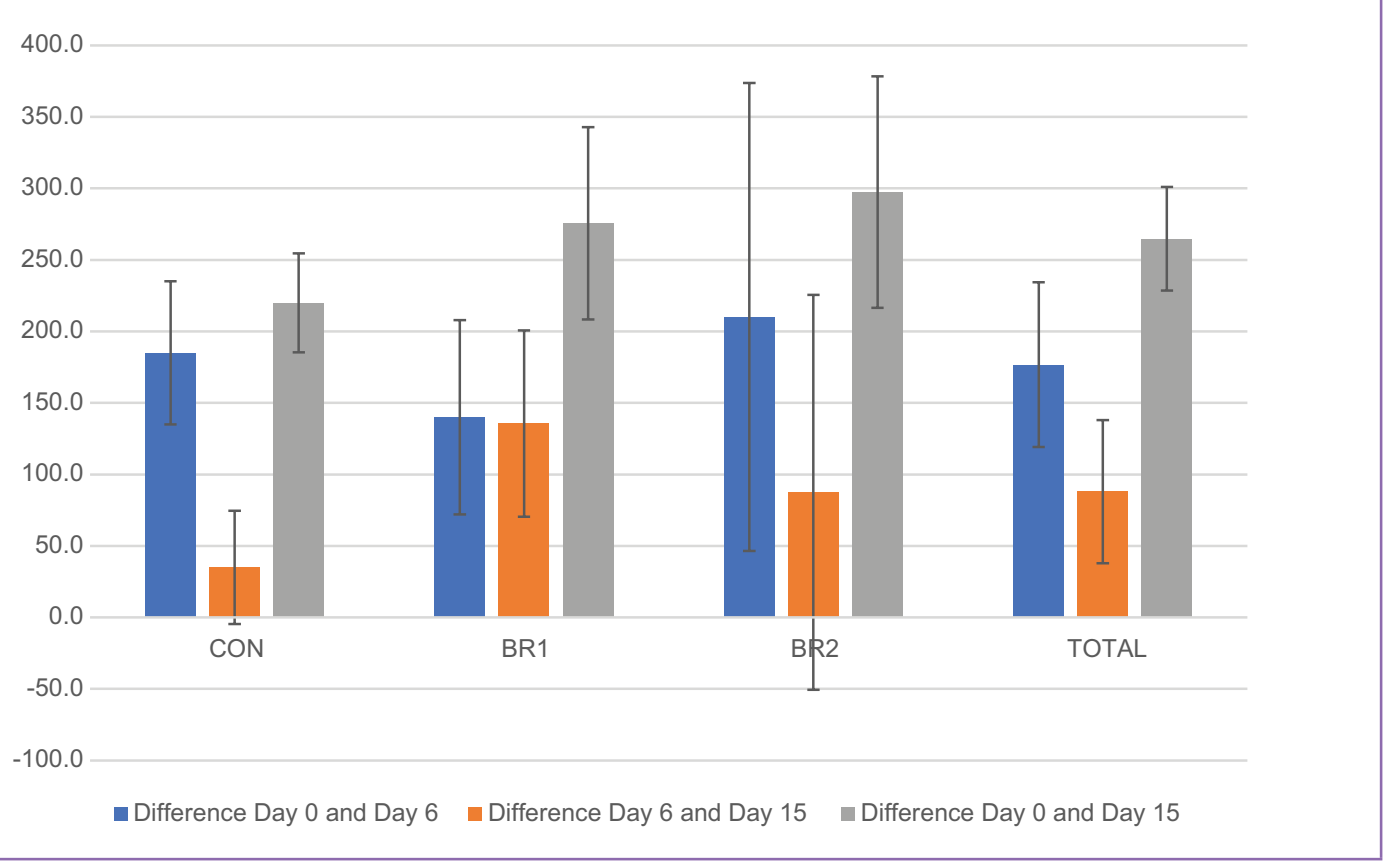

$35.45 \%$ increase for BR2 when compared to the CON group [Table 2].

A one-way ANOVA demonstrated that throughout the experiment, blood pressure was not significantly different at any point $(P>0.05)$ for all three groups. Over the 15 days, there were no observable trends in systolic and diastolic blood pressure readings in any of the groups. All 25 cadets completed the online questionnaire (days 8 and 15). Although cadets were encouraged to consume their respective beverages within $30 \mathrm{~min}$, a majority drank their beverages within 5-8 min. Even though a few $(n=2-3)$ cadets reported periodic Gl symptoms (stomach pain, nausea or vomiting, bloating, constipation or hard stools, and diarrhea or loose stools), no significant differences were observed. Gl symptoms could be 


\section{Melton and Kandiah: Influence of varying doses and duration of dietary nitrates from beetroot juice on sprint performance in U.S. army ROTC cadets: A pilot study}

indicative of several factors completely unrelated to BR. A t-test revealed that there was a significant difference in sprint performance between males and females overall at days 0,6 , and $5(P=0.025$, $P=0.005$, and $P=0.004$, respectively). The differences in distance covered between days 0 and 15 were significantly different between males and females $(P=0.032)$ [Table 3].

Table 3: Overall sprint performance: Males versus females

\begin{tabular}{|l|c|c|} 
Variables & \multicolumn{1}{c}{ Males } & Females \\
\hline Participants & 15 & 10 \\
\hline Day 0 & $1182.7 \pm 104.51^{*}$ & $410.0 \pm 52.78$ \\
\hline Day 6 & $1420.0 \pm 148.58^{* *}$ & $496.0 \pm 51.36$ \\
\hline Day 15 & $1520.0 \pm 125.89^{* *}$ & $566.0 \pm 51.47$ \\
\hline Diff-days 0 and 6 & $237.3 \pm 91.65^{*}$ & $86.0 \pm 31.84$ \\
\hline Diff-days 6 and 15 & $100.0 \pm 82.51$ & $70.0 \pm 27.53$ \\
\hline Diff-days 0 and 15 & $337.3 \pm 49.72 *$ & $156.0 \pm 27.78$ \\
\hline
\end{tabular}

CON: Control group ( $0 \mathrm{mg} \mathrm{NO}{ }_{3}^{-}$), BR1: Low BR group ( $\sim 300 \mathrm{mg}$ $\mathrm{NO}_{3}-$ ), BR2: High BR group ( 600 $\mathrm{mg} \mathrm{NO}_{3}^{-}$). Difference 1 and $2=$ Day 6 sprint distance - Day 0 sprint distance. Difference 2 and $3=$ Day 15 sprint distance - Day 6 sprint distance. Difference 1 and 3 = Day 15 sprint distance - Day 0 sprint distance. Presented as mean \pm SE. $(n=25) .{ }^{*} P<0.05 ; * * P<0.001$
Since differences in men and women cadets overall performance were observed, a one-way ANOVA was performed to determine if a dose-related effect existed between genders. In males, no significant differences in sprint distances were noted in days 0 , 6 , and $15(P>0.05)$. Further, there were no statistical differences between sprints $(P>0.05)$. However, a BR dose-related improvement in performance was observed in all groups of males; over the 15-day timeframe. Performance improvements for CON, BR1, and BR2 were 230, 360, and 392 meters, respectively. When compared to CON-males, this was a $56.52 \%$ improvement for BR1 and $70.43 \%$ for BR2-males [Table 4]. In women, there was only a significant difference between groups for distance covered between days 0 and $6(P=0.033)$, with no other significant differences observed $(P>0.05)$. The dose-related enhancement of BR was not observed in females; the sprint performance of CON females improved by $210 \mathrm{~m}, \mathrm{BR} 1$ by $100 \mathrm{~m}$, and BR2 by $140 \mathrm{~m}$ [Table 4].

A $t$-test revealed that there was a significant difference in the sprint performance of males at days 6 and 15 ( $P=0.012$, and $P=0.033$, respectively). Cadets who were categorized as fit and unfit improved similarly from days 0 to 15 [Table 5]; the

Table 4: Treatment group sprint performance: Males versus females

\begin{tabular}{|c|c|c|c|c|}
\hline Variables & CON & BR1 & BR2 & Total \\
\hline Male & 4 & 6 & 5 & 15 \\
\hline Female & 4 & 3 & 3 & 10 \\
\hline \multicolumn{5}{|c|}{ Male } \\
\hline Day 0 & $840.0 \pm 111.95$ & $1356.7 \pm 95.98$ & $1248.0 \pm 244.34$ & $1182.67 \pm 104.51$ \\
\hline Day 6 & $1060.0 \pm 170.88$ & $1580.0 \pm 97.84$ & $1516.0 \pm 404.99$ & $1420.0 \pm 148.58$ \\
\hline Day 15 & $1070.0 \pm 124.77$ & $1720.0 \pm 154.92$ & $1640.0 \pm 254.87$ & $1520.0 \pm 125.89$ \\
\hline Diff-days 0 and 6 & $220.0 \pm 95.92$ & $223.3 \pm 81.55$ & $268.0 \pm 268.97$ & $237.3 \pm 91.65$ \\
\hline Diff-days 6 and 15 & $10.0 \pm 75.50$ & $140.0 \pm 96.75$ & $124.0 \pm 227.54$ & $100.0 \pm 82.51$ \\
\hline Diff-days 0 and 15 & $230.0 \pm 68.07$ & $363.3 \pm 74.37$ & $392.0 \pm 107.81$ & $337.3 \pm 49.72$ \\
\hline \multicolumn{5}{|c|}{ Female } \\
\hline Day 0 & $430.0 \pm 71.88$ & $453.3 \pm 131.32$ & $1248.0 \pm 244.34$ & $410.0 \pm 52.78$ \\
\hline Day 6 & $580.0 \pm 93.10$ & $426.7 \pm 96.15$ & $453.3 \pm 70.55$ & $496.0 \pm 51.36$ \\
\hline Day 15 & $640.0 \pm 65.32$ & $553.3 \pm 129.79$ & $480.0 \pm 83.27$ & $566.0 \pm 51.47$ \\
\hline Diff-days 0 and 6 & $150.0 \pm 41.23 *$ & $-26.7 \pm 35.28^{*}$ & $113.3 \pm 33.3^{*}$ & $86.0 \pm 31.84$ \\
\hline Diff-days 6 and 15 & $60.0 \pm 34.64$ & $126.7 \pm 63.60$ & $26.7 \pm 48.07$ & $70.0 \pm 27.53$ \\
\hline Diff-days 0 and 15 & $210.0 \pm 30.00$ & $100.0 \pm 60.00$ & $140.0 \pm 50.33$ & $156.0 \pm 27.78$ \\
\hline
\end{tabular}

CON: Control group ( $\sim \mathrm{mg} \mathrm{NO}_{3}-$ ), BR1: Low BR group ( 300 $\mathrm{mg} \mathrm{NO}_{3}^{-}$), BR2: High BR group ( $600 \mathrm{mg} \mathrm{NO}{ }_{3}^{-}$). Difference 1 and $2=$ Day 6 sprint distance - Day 0 sprint distance. Difference 2 and $3=$ Day 15 sprint distance - Day 6 sprint distance. Difference 1 and $3=$ Day 15 sprint distance - Day 0 sprint distance. Presented as mean \pm SE. $(n=25)$. ${ }^{*} P<0.05$ 


\section{Melton and Kandiah: Influence of varying doses and duration of dietary nitrates from beetroot juice on sprint performance in U.S. army ROTC cadets A pilot study}

Table 5: Overall sprint performance: Fit versus unfit cadets

\begin{tabular}{|l|c|c|}
\hline Variables & \multicolumn{1}{c}{ Fit } & Unfit \\
\hline Participants & 10 & 15 \\
\hline Day 0 & $1050.0 \pm 196.85$ & $756.0 \pm 100.48$ \\
\hline Day 6 & $1324.0 \pm 258.85^{*}$ & $868.0 \pm 113.77$ \\
\hline Day 15 & $1364 \pm 239.66^{*}$ & $988.0 \pm 119.71$ \\
\hline Diff-days 0 and 6 & $274.0 \pm 93.43$ & $112.0 \pm 70.70$ \\
\hline Diff-days 6 and 15 & $40.0 \pm 78.66$ & $120.0 \pm 65.76$ \\
\hline Diff-days 0 and 15 & $314.0 \pm 51.99$ & $232.0 \pm 48.90$ \\
\hline
\end{tabular}

CON: Control group ( $0 \mathrm{mg} \mathrm{NO}-$ ), BR1: Low BR group ( $300 \mathrm{mg}$ $\left.\mathrm{NO}_{3}-\right)$, BR2: High BR group ( $\left.600 \mathrm{mg} \mathrm{NO}{ }_{3}^{-}\right)$. Difference 1 and 2: Day 6 sprint distance - Day 0 sprint distance. Difference 2 and $3=$ Day 15 sprint distance - Day 6 sprint distance. Difference 1 and 3 = Day 15 sprint distance - Day 0 sprint distance. Presented as mean \pm SE. $(n=25) .{ }^{*} p<0.05$

difference in improvements was insignificant $(P=$ $0.877)$. This is an important observation to note, given there is evidence that $B R$ enhances low-fit but not high-fit male runners. ${ }^{[20]} A t$-test was used to assess differences in performance between fit and unfit cadets by the treatment group. Between fit and unfit cadets, there were no significant differences between treatment groups in distances completed on all three sprints or in differences between sprints [Table 6]. Within fit cadets, BR juice enhanced sprint performance, but this enhancement was not in a linear dose-response related manner. Over the 15 days, improvement in sprint performance was observed in the fit categories for the CON, BR1, and $\operatorname{BR} 2$ (210, 400, and 367 meters, respectively); this shows that when compared to the CON group, there was $90.47 \%$ and a $74.76 \%$ improvement in BR1 and BR2 groups, respectively. Within unfit cadets, BR did not improve performance. Over the 15 days, the unfit cadets in the CON, BR1, and BR3 groups had an observed improvement in sprint performance (230, 213, and 256 meters, respectively). Compared to the CON group, BR1 had a $7.39 \%$ decrease while BR2 had an $11.30 \%$ increase.

\section{Discussion}

At baseline (day 0), there were no differences between treatment groups, despite the three groups having a wide variability of aerobic fitness and body compositions. Due to the disparity among cadets, the Yo-Yo IR1 test was appropriate. The body composition differences observed were similar to what Wennblom and Brechue ${ }^{[21]}$ observed in cadets from the United States Military Academy who ranged in body fat from $6 \%$ to $26 \%$; however, there was a larger range, $4-35 \%$, for the cadets in this study. The body composition of the cadets in the present research was similar to the 13 cadets from a midsize, Midwestern University's ROTC program that Steed et al. ${ }^{[15]}$ evaluated. In that study, males had an average body mass index (BMI) of 24.6 and an average body fat percentage of $11.9 \%$; females had an average BMI of 24.5, and an average body fat percentage of $27.1 \%$. The male cadets in our study had an average BMI and body fat percentage of 24.9 and $14.9 \%$, while females had 25.1 and $26.4 \%$, respectively. Not only were the groups within this study similar to each other but also they were consistent with trends reported in military personnel, and may be representative of the overall military population.

While the sprint tests indicated no statistically significant effect of BR dietary nitrates on sprint performance, a dose-related improvement of BR was observed with a larger difference in distance covered between the first and third sprints observed in the BR2 group ( $297 \mathrm{~m}$ ) compared with the BR1 and CON groups ( $275 \mathrm{~m}$ and $\sim 220 \mathrm{~m}$, respectively). In this small sample, when compared to the CON group, BR2 improved overall performance by $35 \%$ and BR1 improved performance by $25 \%$. Although, the administration of BR1 improved cadets' performance, the improvement was not found to be statistically significant. Observations in sprint performance in males were evident during consumption of varying does of BR which was not prevalent in females. Although the cadets were uninformed by the investigators that BR is supposed to benefit performance, they could have had an inclination, as the BR canister had the label Beet Performer and if this had triggered their curiosity it is possible they might have googled and read about the beverage. However, none of the cadets questioned the researchers about the products they consumed. Given a larger sample, it is possible that these results would have been magnified and a trend toward statistical significance may have been noted. Wylie et al. ${ }^{[9]}$ observed that recreationally active men given $490 \mathrm{ml}$ of concentrated BR over $30 \mathrm{~h}$ before completing the Yo-Yo IR1 test improved by $4.2 \%$ compared to PL $(1704 \pm 304 \mathrm{~m}$ with BR, $1636 \pm 288 \mathrm{~m}$ with $\mathrm{PL}, P<0.05)$. The research conducted by Wylie et al. ${ }^{[9]}$ looked at dosing of nitrate from BR over a $24 \mathrm{~h}$ period. The present study looked at BR consumption consecutively over

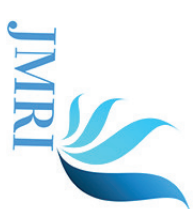




\section{Melton and Kandiah: Influence of varying doses and duration of dietary nitrates from beetroot juice on sprint performance in U.S. army ROTC cadets: A pilot study}

Table 6: Treatment group sprint performance: Fit versus unfit cadets

\begin{tabular}{|c|c|c|c|c|}
\hline Variables & CON & BR1 & BR2 & Total \\
\hline FIT & 4 & 3 & 3 & 10 \\
\hline UNFIT & 4 & 6 & 5 & 15 \\
\hline \multicolumn{5}{|c|}{ FIT } \\
\hline Day 0 & $790.0 \pm 156.10$ & $1133.3 \pm 468.95$ & $1313.3 \pm 478.93$ & $1050.0 \pm 196.85$ \\
\hline Day 6 & $990.0 \pm 215.02$ & $1280.0 \pm 522.05$ & $1813.3 \pm 667.07$ & $1324.0 \pm 258.85$ \\
\hline Day 15 & $1000.0 \pm 165.73$ & $1533.3 \pm 588.48$ & $1680.0 \pm 554.74$ & $1364 \pm 239.66$ \\
\hline Diff-days 0 and 6 & $200.0 \pm 96.61$ & $146.7 \pm 53.30$ & $500.0 \pm 268.58$ & $274.0 \pm 93.43$ \\
\hline Diff-days 6 and 15 & $10.0 \pm 75.50$ & $253.3 \pm 66.67$ & $-133.3 \pm 196.41$ & $40.0 \pm 78.66$ \\
\hline Diff-days 0 and 15 & $210.0 \pm 57.45$ & $400.0 \pm 120.00$ & $366.7 \pm 85.11$ & $314.0 \pm 51.99$ \\
\hline \multicolumn{5}{|c|}{ UNFIT } \\
\hline Day 0 & $480.0 \pm 73.03$ & $1016.7 \pm 148.90$ & $664.0 \pm 178.37$ & $756.0 \pm 100.48$ \\
\hline Day 6 & $650.0 \pm 103.76$ & $1153.0 \pm 211.01$ & $700.0 \pm 147.24$ & $868.0 \pm 113.77$ \\
\hline Day 15 & $710.0 \pm 95.74$ & $1230.0 \pm 206.67$ & $920.0 \pm 216.15$ & $988.0 \pm 119.71$ \\
\hline Diff-days 0 and 6 & $170.0 \pm 47.26$ & $136.7 \pm 102.43$ & $36.0 \pm 181.92$ & $112.0 \pm 70.70$ \\
\hline Diff-days 6 and 15 & $60.0 \pm 34.64$ & $76.7 \pm 84.92$ & $220.0 \pm 173.44$ & $120.0 \pm 65.76$ \\
\hline Diff-days 0 and 15 & $230.0 \pm 47.26$ & $213.3 \pm 75.31$ & $256.0 \pm 122.54$ & $232.0 \pm 48.90$ \\
\hline
\end{tabular}

CON: Control group ( $\sim \mathrm{mg} \mathrm{NO}_{3}^{-}$), BR1: Low BR group ( 300 $\mathrm{mg} \mathrm{NO}_{3}^{-}$), BR2 = high BR group ( 600 $\mathrm{mg} \mathrm{NO}_{3}^{-}$). Difference 1 and $2=$ Day 6 sprint distance - Day 0 sprint distance. Difference 2 and $3=$ Day 15 sprint distance - Day 6 sprint distance. Difference 1 and 3 = Day 15 sprint distance - Day 0 sprint distance. Presented as mean \pm SE. $(n=25)$

6 and 15 days, respectively. According to Wylie et al., ${ }^{[22]}$ BR may help with shorter repeated sprints with short recovery periods, such as the Yo-Yo IR1, but may not help with longer repeated sprints with longer recovery periods. Anaerobic conditions at maximal exertion in repeated sprints with little recovery would be more likely to convert $\mathrm{NO}_{2}$ - to NO, such as in hypoxia or high-altitude where Bakker et al. ${ }^{[23]}$ observed that BR improved arterial endothelial and reduced muscle metabolic distress during exercise.

Between cadets, a difference was observed between male and female participants. The BR seemed to enhance male sprint performance, while having no effect on female sprint performance. In males, there was a dose related improvement with BR use; BR1 improved by $57 \%$ and BR2 improved by $70 \%$ compared to the CON group. A possible speculation of improved sprint performance in males could be due to greater compliance in adhering to the prescribed dietary protocol, lesser incidence of side effects (e.g., pain, bloating, Gl symptoms, and lack of menstrual issues). In addition, since much of the participants' information was selfreported (e.g., alcohol, frequency, and intensity of the workout) there could be have some erroneous reporting of information by female participants.
A possible factor that cannot be negated is the influence of female hormonal factors in the metabolism of dietary nitrates from BR. It was observed that BR improved performance in cadets categorized as fit, but not unfit cadets. In fit cadets, the improvement was not dose-related; BR1 improved by $90 \%$ and BR2 improved by $75 \%$ compared to the CON group. Carriker et al. ${ }^{[20]}$ found contradictory results; BR was associated with improved performance in low-fit male runners but not high-fit. Since this study lasted 2 weeks, it is likely that those cadets of higher fitness levels exercised on their own apart from daily ROTC conditioning and experienced volumetric enhancements.

The findings of the present research are not in congruence with other studies that looked at BR supplementation for 6 days, where significance was noted. ${ }^{[24,25]}$ In the present research, at day 6, all treatment groups were similar and no benefit was found with either a single or double dose of BR. Many factors affect sprint performance, such as sleep, ${ }^{[26]}$ fatigue and substrate availability, ${ }^{[27]}$ caffeine, ${ }^{[28]}$ nutrition and diet, ${ }^{[29]}$ and pain, bloating, or other GI symptoms, and/or menstrual issues. ${ }^{[30,31]}$ It is possible that had these factors been tightly controlled in that the cadets were not free-living but residing in a confined environment, differences 


\section{Melton and Kandiah: Influence of varying doses and duration of dietary nitrates from beetroot juice on sprint performance in U.S. army ROTC cadets A pilot study}

in sprint performance could have been realized. . $^{\text {[0-34] }}$ Based on the results since very few participants reported experiencing minor Gl problems military personnel, especially ROTC Cadets, would likely benefit if they were able to consume BR for a longer duration thereby increasing adaptability to a higher concentration of nitrates with increased performance outcomes. The largest improvement observed in this study was with cadets who scored in the fit category. In addition, a single dose of BR was just as beneficial as double doses. Although when analyzing by gender, men benefited more from a double dose than that of a single dose of $B R$. A speculation of this could be due to variance in anthropometrics that are common between the two genders. These results would indicate that more studies are warranted to evaluate the efficacy of BR as a performance supplement in females.

There are several limitations to this study that may account for the unexpected results. ${ }^{[32-34]}$ First, there was no blinding in this experiment and future studies should consider various study designs. Second, the participants were not incentivized to participate except for the complementary BOD POD and predictive $\mathrm{VO}_{2 \max }$ assessments and the option to miss morning PT. Without offering the participants an incentive, they may have disregarded the researcher's requests and dietary guidelines for the study. Third, the participants had no real practice run through and some of the increases seen between day 0 and day 6 is directly related to familiarization with the testing protocol. Fourth, for randomization, the study used predicted $\mathrm{VO}_{2 \max }$ relative for age and sex as the determinate of cardiovascular fitness. The predicted $\mathrm{VO}_{2 \max }$ is much easier to administer and requires less time or involvement from the participant, but it is not as accurate as the full $\mathrm{VO}_{2 \max }$ test. Fifth, the researchers did not collect baseline blood pressure from the cadets. Sixth, the menstrual cycle for the female cadets (e.g., early follicular phase vs. luteal phase) was not assessed. Finally, the participants selfreported their alcohol intake, dietary compliance, workout frequency and intensity, and GI symptoms experienced.

\section{References}

1. Conner TS, Brookie KL, Carr AC, Mainvil LA, Vissers MC. Let them eat fruit! The effect of fruit and vegetable consumption on psychological well-being in young adults: A randomized controlled trial. PLoS One 2017;12:e0171206.
2. Thompson D, Ferry RJ Jr, Cullen KW, Liu Y. Improvement in fruit and vegetable consumption associated with more favorable energy density and nutrient and food group intake, but not kilocalories. J Acad Nutr Diet 2016;116:1443-9.

3. Schwingshackl L, Hoffmann G, Kalle-Uhlmann T, Arregui M, Buijsse B, Boeing $\mathrm{H}$. Fruit and vegetable consumption and changes in anthropometric variables in adult populations: A systematic review and meta-analysis of prospective cohort studies. PLoS One 2015;10:e0140846.

4. Coleman E. Reap the benefits of beet root juice evidence suggests it improves heart health and athletic performance. Todays Diet 2012;12:48.

5. Hertzler S. Nitrate supplementation for cardiovascular health and exercise performance. SCANs Pulse 2012;31:7-11.

6. Hord NG, Tang Y, Bryan NS. Food sources of nitrates and nitrites: The physiologic context for potential health benefits. Am J Clin Nutr 2009;90:1.

7. Van Duyn MA, Pivonka E. Overview of the health benefits of fruit and vegetable consumption for the dietetics professional: Selected literature. J Am Diet Assoc 2000;100:1511-21.

8. Collofello B, Moskalik B, Essick E. Acute dietary nitrate supplementation decreases systolic blood pressure and increases dry static apnea performance in females. J Exerc Physiol Online 2014;17:15.

9. Wylie LJ, Kelly J, Bailey SJ, Blackwell JR, Skiba PF, Winyard PG, et al. Beetroot juice and exercise: Pharmacodynamic and dose-response relationships. J Appl Physiol (1985) 2013;115:325-36.

10. Kisaalita NR, Robinson ME. Attitudes and motivations of competitive cyclists regarding use of banned and legal performance enhancers. J Sports Sci Med 2014;13:44-50.

11. Ford K, Glymour C. The enhanced warfighter. Bull Atomic Sci 2014;70:43-53.

12. Cermak NM, Gibala MJ, van Loon LJ. Nitrate supplementation's improvement of $10-\mathrm{km}$ timetrial performance in trained cyclists. Int J Sport Nutr Exerc Metab 2012;22:64-71.

13. Murphy M, Eliot K, Heuertz RM, Weiss E. Whole beetroot consumption acutely improves running performance. J Acad Nutr Diet 2012;112:548-52.

14. Hoon MW, Jones $A M$, Johnson NA, Blackwell JR, Broad EM, Lundy B, et al. The effect of variable doses of inorganic nitrate-rich beetroot juice on simulated 2,000-m rowing performance in trained athletes. Int J Sports Physiol Perform 2014;9:615-20.

15. Steed CL, Krull BR, Morgan AL, Tucker RM, Ludy MJ. Relationship between body fat and physical fitness in army ROTC cadets. Mil Med 2016;181:1007-12.

16. Bescós R, Sureda A, Tur JA, Pons A. The effect of nitric-oxide-related supplements on human performance. Sports Med 2012;42:99-117.

17. Webb AJ, Patel N, Loukogeorgakis S, Okorie M,

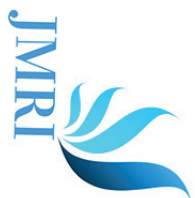


Aboud Z, Misra S, et al. Acute blood pressure lowering, vasoprotective, and antiplatelet properties of dietary nitrate via bioconversion to nitrite. Hypertension 2008;51:784-90.

18. Krustrup P, Mohr M, Amstrup T, Rysgaard T, Johansen J, Steensberg A, et al. The yo-yo intermittent recovery test: Physiological response, reliability, and validity. Med Sci Sports Exerc 2003;35:697-705.

19. Bangsbo J, laia FM, Krustrup P. The Yo-Yo intermittent recovery test: A useful tool for evaluation of physical performance in intermittent sports. Sports Med 2008;38:37-51.

20. Carriker CR, Vaughan RA, VanDusseldorp TA, Johnson KE, Beltz NM, McCormick JJ, et al. Nitrate-containing beetroot juice reduces oxygen consumption during submaximal exercise in low but not high aerobically fit male runners. J Exerc Nutrition Biochem 2016;20:27-34.

21. Wennblom ME, Brechue, WF. Fat mass: Accumulation and distribution in athletes and military personnel. Med Sci Sports Exerc 2012;44:801.

22. Wylie LJ, Bailey SJ, Kelly J, Blackwell JR, Vanhatalo A, Jones AM. Influence of beetroot juice supplementation on intermittent exercise performance. Eur J Appl Physiol 2016;116:415-25.

23. Bakker $E$, Engan $H$, Patrician A, Schagatay $E$, Karlsen $\mathrm{T}$, Wisløff $\mathrm{U}$, et al. Acute dietary nitrate supplementation improves arterial endothelial function at high altitude: A double-blinded randomized controlled cross over study. Nitric Oxide 2015;50:58-64.

24. Bailey SJ, Winyard P, Vanhatalo A, Blackwell JR, Dimenna FJ, Wilkerson DP, et al. Dietary nitrate supplementation reduces the $\mathrm{O}_{2}$ cost of lowintensity exercise and enhances tolerance to highintensity exercise in humans. J Appl Physiol (1985) 2009;107:1144-55.

25. Bailey SJ, Winyard PG, Vanhatalo A, Blackwell JR, DiMenna FJ, Wilkerson DP, et al. Acute L-arginine supplementation reduces the $\mathrm{O}_{2}$ cost of moderate-intensity exercise and enhances highintensity exercise tolerance. J Appl Physiol (1985)
2010;109:1394-403.

26. Abedelmalek S, Souissi N, Chtourou H, Denguezli M, Aouichaoui C, Ajina $M$, et al. Effects of partial sleep deprivation on proinflammatory cytokines, growth hormone, and steroid hormone concentrations during repeated brief sprint interval exercise. Chronobiol Int 2013;30:502-9.

27. Waldron $\mathrm{M}$, Highton J. Fatigue and pacing in highintensity intermittent team sport: An update. Sports Med 2014;44:1645-58.

28. Mohr M, Nielsen JJ, Bangsbo J. Caffeine intake improves intense intermittent exercise performance and reduces muscle interstitial potassium accumulation. J Appl Physiol (1985) 2011;111:1372-9.

29. Couto PG, Bertuzzi R, de Souza CC, Lima HM, Kiss M, De-Oliveira FR, et al. High carbohydrate diet induces faster final sprint and overall 10,000m times of young runners. Pediatr Exerc Sci 2015;27:355-63.

30. de Oliveira EP, Burini RC. Carbohydrate-dependent, exercise-induced gastrointestinal distress. Nutrients 2014;6:4191-9.

31. Botcazou M, Gratas-Delamarche A, Allain S, Jacob C, Bentué-Ferrer $D$, Delamarche $P$, et al. Influence of menstrual cycle phase on catecholamine response to sprint exercise in the woman. Appl Physiol Nutr Metab 2006;31:604-11.

32. Coles LT, Clifton PM. Effect of beetroot juice on lowering blood pressure in free-living, disease-free adults: A randomized, placebo-controlled trial. Nutr J 2012;11:106.

33. Vanhatalo A, Bailey SJ, Blackwell JR, DiMenna FJ, Pavey TG, Wilkerson DP, et al. Acute and chronic effects of dietary nitrate supplementation on blood pressure and the physiological responses to moderateintensity and incremental exercise. Am J Physiol Regul Integr Comp Physiol 2010;299:R1121-31.

34. Jajja A, Sutyarjoko A, Lara J, Rennie K, Brandt K, Qadir O, et al. Beetroot supplementation lowers daily systolic blood pressure in older, overweight subjects. Nutr Res 2014;34:868-75. 